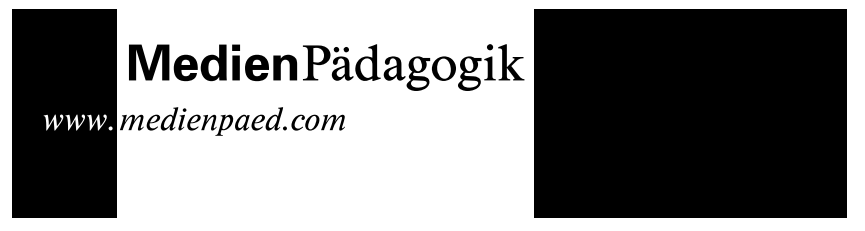

Gabi Reinmann-Rothmeier

30.10 .2002

\section{Mediendidaktik und Wissensmanagement}

Mediendidaktik und Wissensmanagement - das ist auf den ersten Blick ein ungleiches Paar: Die Mediendidaktik ist eine Teildisziplin der (Medien-) Pädagogik, während Wissensmanagement ein genuin wirtschaftliches Thema mit betriebswirtschaftlichen Akzenten ist. Annäherungen zwischen der Mediendidaktik und Wissensmanagement gibt es zum einen durch den sog. E-Learning-Trend seitens der Wirtschaft, der mediendidaktisches Wissen und Können auf den Plan ruft, zum anderen durch wachsendes Interesse am Thema Wissensmanagement seitens der Pädagogik. Der Beitrag beschreibt sowohl für das E-Learning als auch für das Wissensmanagement aus pädagogisch-psychologischer Sicht jeweils ein Orientierungsmodell; beide Modelle machen die Berührungspunkte zwischen Mediendidaktik und Wissensmanagement deutlich. Neben einem Überblick über wirtschaftliche Argumente für eine Verschmelzung von E-Learning und Wissensmanagement werden aufbauend auf den beiden Orientierungsmodellen theoretische und praktische Verbindungslinien zwischen ELearning und Wissensmanagement sowie die Rolle der Mediendidaktik in dem daraus entstehenden Wechselverhältnis herausgearbeitet.

\section{Mediendidaktik und Wissensmanagement - ein ungleiches Paar}

In den vergangenen Jahren sind - u. a. im Zuge der neuen Medien - die Berührungsängste zwischen Wirtschaft und Pädagogik kleiner geworden. Grosse Initiativen wie «Schulen ans Netz», die Förderung von Modellprojekten durch wirtschaftsnahe Stiftungen oder regionalen Arbeitsgemeinschaften, in denen Vertreter aus Wirtschaft und Bildung zusammenarbeiten, zeugen davon, dass man Annäherung sucht und an manchen Stellen auch findet. Dennoch kann nicht von einer flächendeckenden Of- fenheit die Rede sein, wenn es darum geht, in der Medienfrage seitens der Wirtschaft auf die Pädagogik oder seitens der Pädagogik auf die Wirtschaft zuzugehen. Das zeigt sich besonders deutlich in der Praxis des Lehrens und Lernens, die den Verlautbarungen zur Zukunft der Bildung mit neuen Medien chronisch hinterherhinkt. Wenn in diesem Beitrag die «Mediendidaktik» dem «Wissensmanagement» gegenübergestellt werden soll, haben wir ein exemplarisches Beispiel für das Spannungsverhältnis zwischen Wirtschaft und Pädagogik. Worauf dieses Spannungsverhältnis zurückzuführen ist, zeigt zunächst einmal eine genauere Analyse der beiden Begrifflichkeiten.

\subsection{Mediendidaktik}

Die Mediendidaktik gilt als Teilbereich der Didaktik; letztere wiederum ist eine pädagogische Teildisziplin, die sich mit der Theorie und Praxis des Lehrens und Lernens beschäftigt (vgl. Issing, 2002). Zu den Aufgaben der Didaktik gehört es, Ziele, Inhalte, Strategien und Methoden des Lehrens und Lernens zu beschreiben, aber auch normativ vorzugeben, sie zu analysieren, zu erforschen und für konkrete Bildungssituationen zu planen und zu evaluieren. Die Didaktik hat eine lange Tradition und es gibt eine Vielzahl unterschiedlicher didaktischer Theorien (z. B. Kron, 1993). Auch der Mediendidaktik wird mit Verweis auf Comenius und dessen Plädoyer für das Bild eine traditionsreiche Wurzel nachgesagt. Das ändert allerdings nichts an der Tatsache, dass man sich angesichts der rasanten Entwicklung auf dem Medienmarkt in der Praxis eher schwer tut, didaktische Fragen insbesondere zu den nеиеn Medien systematisch mit didaktischen Theorien zu verbinden.

Im Rahmen der Medienpädagogik hatte und hat die Mediendidaktik bisweilen mit dem Vorwurf zu kämpfen, es handle sich um eine «blosse» Technologie, wobei man vor allem Modelle des Instructional Design im Blick hat (vgl. Schott, 1991). Auch die Reduktion der Mediendidaktik auf das Ziel, eine informationstechnische Grundbildung zu fördern, hat nicht gerade dazu beigetragen, den Stellenwert der Mediendidaktik innerhalb der Medienpädagogik zu stärken. Ansätze zur Verbindung mediendidaktischer Bemühungen mit Zielen der Medienkompetenzförderung dagegen zeigen, dass gerade in der Praxis des Lehrens und Lernens der wohl sinnvollste Weg darin besteht, medienerzieherische und mediendidaktische Konzepte aneinander zu koppeln statt sie gegeneinander auszuspielen (Aufenanger, 1999). 
Lange Zeit hat sich die Mediendidaktik auf Überlegungen und Empfehlungen konzentriert, wie man Medien für verschiedene Lehr-Lernsituationen auswählt, kombiniert, einsetzt und bewertet. Man könnte das mit einer Art Legespiel vergleichen, bei dem man Karten, Figuren oder andere Gegenstände hin- und herschiebt, verschiedene Muster ausprobiert, wieder zerlegt und neu zusammensetzt. Neuere Ansätze der Mediendidaktik ergänzen dieses «Legespiel» durch ein «Konstruktionsspiel», indem sie auch die Herstellung und Gestaltung von Medien/Medienprodukten zur mediendidaktischen Aufgabe macht (z. B. Kerres, 2001). Hier ist eindeutig eine Weiterentwicklung der Mediendidaktik zu beobachten, die vor allem durch die Lehr-Lernpotentiale der neuen Medien motiviert ist.

Der primäre «Ort» der Mediendidaktik ist die Pädagogik: Auf der Grundlage pädagogischer Zielsetzungen hat es sich die Mediendidaktik zur Aufgabe gemacht, mit dem Einsatz und der Gestaltung von Medien das Lehren und Lernen in verschiedenen Bildungssituationen (Schule, Hochschule, Arbeitsplatz, Freizeit etc.) zu verbessern.

1.2 Wissensmanagement

Anders als die Mediendidaktik ist Wissensmanagement keine genuin wissenschaftliche Teildisziplin, sondern das Ergebnis informationstechnischer und betriebswirtschaftlicher Bemühungen, Lösungsansätze für ökonomische, aber auch gesellschaftliche Phänomene zu finden wie Informationsflut, wachsende Geschwindigkeit von Informationsgenerierung und verbreitung, steigende Vernetztheit und Komplexität von Wissen u. a. (z. B. North, 1998). Zwar gibt es Stimmen, die dem Wissensmanagement nach wie vor nur den Status eines Modetrends in der Managementlehre zubilligen. Die wachsende Anzahl wissenschaftlicher Beiträge zu diesem Thema sowie die Aufnahme des Begriffs «Wissensmanagement» in die Bezeichnung etwa von Lehrstühlen oder Studiengängen zeigt jedoch, dass sich das Thema den Rang eines wissenschaftlich ernst zu nehmenden Faches erworben hat.

Relativ früh forderten Vertreter der Wissenschaft, Mensch, Organisation und Technik als die drei zentralen Komponenten des Wissensmanagement zu etablieren (Bullinger, Wörner \& Prieto, 1998). Entscheidend hierfür war und ist die Auffassung von Wissen als einem personen- und kontextabhängigen Gut, das anders als Information nicht ohne die Wissensträger zu «managen» ist. Als Ziel des Wissensmanagements gilt gemeinhin nicht nur
Information, sondern Wissen im genannten Sinne sowie Kompetenzen und Erfahrungen in Organisationen systematisch zu erfassen, zu bewahren und zu nutzen, um auf diesem Wege die Wissensbasis, Reaktionsfähigkeit und Effizienz der Organisation zu verbessern. Dabei spielt die technische Informations- und Kommunikationsinfrastruktur sowohl theoretisch als auch praktisch eine tragende Rolle.

Wahrscheinlich gibt es inzwischen genauso viele WissensmanagementModelle wie es didaktische Theorien (s. o.) gibt: Sehr viele dieser Modelle versuchen, die genannten Ziele mit vorrangig betriebswirtschaftlichen Methoden zu erreichen (z. B. Probst, Raub \& Romhardt, 2000); vereinzelt findet man Ansätze, die lernpsychologische Erkenntnisse miteinbeziehen (z. B. Schüppel, 1996) und/oder soziologische Aspekte und Theorien einbinden (z. B. Willke, 1998). Pädagogisch-psychologische Modelle mit Wirkung auf den ökonomisch orientierten Nutzerkreis gab und gibt es im Wissensmanagement kaum (siehe Abschnitt 3).

Der primäre «Ort» des Wissensmanagements ist die Wirtschaft: Auf der Grundlage ökonomischer Zielsetzungen hat es sich das Wissensmanagement zur Aufgabe gemacht, mit der Ressource Wissen bewusst, systematisch und geplant umzugehen, um die aktuelle Wettbewerbssituation und die Reaktions- und Lernfähigkeit von Organisationen zu erhöhen.

1.3 Mediendidaktik meets Wissensmanagement: Erste Annäherungen

Sieht man sich die Herkunft, die wesentlichen Merkmale und Ziele der Mediendidaktik einerseits und des Wissensmanagements andererseits genauer an, verwundert es nicht, dass es schwierig ist, die beiden Begriffe mit ihren zugrundeliegenden Auffassungen und Konzepten zusammenzubringen. Und doch gibt es - wiederum im Zuge der Entwicklungen auf dem Sektor der neuen Medien - erste Annäherungen sowohl von Seiten der Wirtschaft als auch von Seiten der Pädagogik.

Im wirtschaftlichen Kontext mehren sich die Stimmen, die für eine Einbindung mediendidaktischer Erkenntnisse und Ansätze plädieren, wenn es um das sog. E-Learning geht. Die gesamte E-Learning-Szene, aber auch vermehrte Bemühungen, E-Learning an das Wissensmanagement oder umgekehrt zu koppeln (siehe Abschnitt 4), werfen die Frage auf, wie man medienbasierte Wissens- und Arbeitsumgebungen lernförderlich gestalten kann (z. B. Back, Bendel \& Stoller-Schai, 2001). Erschwerend auf eine 
Beantwortung dieser Frage wirkt sich aus, dass der Begriff des E-Learning im wirtschaftlichen Kontext sehr heterogen verwendet wird und eine explizite Anbindung an pädagogisch-psychologische und didaktische LehrLernkonzepte fehlt. Vor diesem Hintergrund soll in Abschnitt 2 ein Orientierungsmodell zum E-Learning vorgestellt werden, das die genannten Schwierigkeiten mindern hilft.

Im pädagogischen Kontext wächst das Interesse an Fragen des organisationalen Wandelns durch Medien im Allgemeinen (z. B. SchulzZander, 2001) sowie an Möglichkeiten des Wissensmanagements in Schule (z. B. Reinmann-Rothmeier \& Mandl, 1997) und Hochschule (z. B. MüllerBöling, 2001) im Besonderen. Zwar gibt es nach wie vor (berechtigte) Bedenken, den Managementbegriff im Rahmen bildungsbezogener Problemstellungen aufzunehmen; trotzdem wächst die Aufmerksamkeit für neue Möglichkeiten der Strukturierung, Weitergabe und Umsetzung von Wissen mit Hilfe der neuen Medien - und hier bieten Wissensmanagement-Modelle eine Reihe fruchtbarer Impulse auch für pädagogische Fragen. Wie bereits erwähnt, sind die defizitäre Einbindung psychologischer Momente beim «Managen» von Wissen und die Dominanz betriebswirtschaftlicher und informationstechnischer Lösungsansätze im Wissensmanagement nach wie vor Hindernisse für eine praktische Umsetzung von Wissensmanagement in Bildungskontexten. Vor diesem Hintergrund soll in Abschnitt 3 ein heuristisches Wissensmanagement-Modell vorgestellt werden, das diese Defizite auffängt.

Aus dem bisher Gesagten ergibt sich ein Beeinflussungsverhältnis von der Wirtschaft auf die Pädagogik über das E-Learning sowie von der Pädagogik auf die Wirtschaft über eine pädagogisch-psychologische Auffassung von Wissensmanagement. Wie ein «Mediendidaktik meets Wissensmanagement» angesichts dieser Überlegungen konkret aussehen kann, soll in Abschnitt 4 näher dargestellt werden: Hier kann gezeigt werden, wie sich verschiedene Wissensprozesse aus der Perspektive des Wissensmanagements mit verschiedenen E-Learning-Varianten aus mediendidaktischer Perspektive kombinieren lassen.

Die nachfolgende Abbildung 1 veranschaulicht noch einmal das skizzierte Argumentationsmuster:

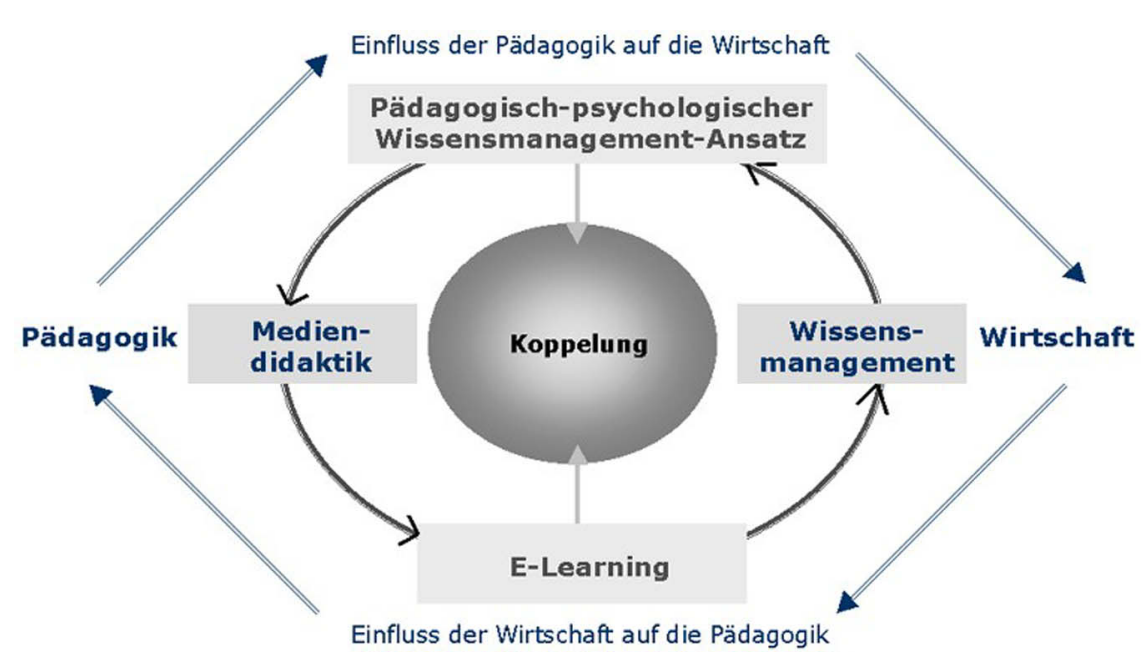

Abb. 1: Wissensmanagement meets Mediendidaktik

\section{Orientierungsmodell zum E-Learning}

\subsection{Der Begriff «E-Learning»}

E-Learning heisst zunächst einmal nichts anderes als «electronic learning», also ein Lernen mit neuen Medien. Durch was sich die neuen Medien auszeichnen, ist inzwischen fast schon Allgemeingut (vgl. Issing \& Klimsa, 2002):

- Da ist zum einen das Potential der Multimedialität: Damit ist die Möglichkeit gemeint, Information und Wissen durch die Integration verschiedener Medien und verschiedener Symbolsysteme darzustellen.

- Da ist zum Zweiten das Potential der Interaktivität: Damit ist die Möglichkeit gemeint, als Mediennutzer mit dem medialen System zu interagieren und unmittelbare Rückmeldung vom Medium zu erhalten.

- Und da ist zum Dritten das Potential der Vernetzung: Damit ist die Möglichkeit gemeint, mit Hilfe der neuen Medien auch neue Kommunikationsformen zu praktizieren, Ort und Zeit durch organisationsinterne wie auch weltweite Vernetzung von Computern zu überwinden.

Wenn man sich diese Haupteigenschaften der neuen Medien vor Augen hält und sich dann überlegt, was E-Learning alles bedeuten kann, wird nachvollziehbar, dass das Feld «E-Learning» unüberschaubar und die Diskussion darüber zwangsläufig vage ist, wenn man nicht klärt, wovon 
man genau spricht. Da ein additives Aufzählen verschiedener Formen des Lernens mit neuen Medien für Fragen der Mediendidaktik wenig hilfreich ist, sollte man ein Orientierungsmodell haben, das einen Überblick im ELearning-Dschungel und damit auch Ansatzpunkte für mediendidaktisches Handeln liefert.

\subsection{Drei «E-Learning»-Varianten}

Eine Möglichkeit für die Entwicklung eines Orientierungsmodells zum ELearning besteht darin, in Anlehnung an Back, Seufert und Kramhöller (1998) drei Leitfunktionen zu unterscheiden, die die neuen Medien auf der Grundlage ihrer Merkmale übernehmen können:

- Wenn jemand im Internet gezielt nach bestimmten Informationsquellen sucht, um ein Problem zu lösen oder im Rahmen eines Weiterbildungsangebots selbstständig elektronisch zugesandtes Material durcharbeitet, liegt eine Form des E-Learning vor. Die Funktion der neuen Medien bei dieser Form des E-Learning besteht darin, lernrelevante Information zu verteilen bzw. zu distribuieren. Man könnte also auch von einem $E$ Learning by distributing sprechen: Die neuen Medien übernehmen hier die Funktion der Distribution von Information.

Aus der Sicht des Lerners besteht diese Form des E-Learning darin, elektronische Information aufzunehmen, selbstgesteuert zu verarbeiten und umzusetzen; ein Lehrender im klassischen Sinne ist für das ELearning by distributing nicht erforderlich.

- Wenn jemand ein Computer Based Training (CBT) für die Bedienung einer neuen Software absolviert oder im Rahmen eines tutoriell begleiteten Web Based Trainings (WBTs) Sprachkenntnisse für einen Auslandsaufenthalt auffrischt, liegt eine weitere Form des E-Learning vor. Die Funktion der neuen Medien bei dieser Form des E-Learning besteht darin, didaktisch aufbereitete Informationen anzubieten, sodass der Lerner sich (weitgehend) ohne personelle Hilfe durch die Interaktion mit dem technischen System neue Inhalte erarbeiten kann. Man könnte also auch von einem E-Learning by interacting sprechen; die neuen Medien übernehmen hier die Funktion, eine Interaktion zwischen Nutzer und System zu ermöglichen.
Aus der Sicht des Lerners besteht diese Form des E-Learning darin, lernrelevante Informationen technisch angeleitet $\mathrm{zu}$ verarbeiten und angebotene Übungen oder Spiele selbstorganisiert durchzuführen. Auch für das E-Learning by interacting ist ein Lehrender im klassischen Sinne nicht erforderlich; möglich, aber nicht zwingend wäre ein Lernberater oder Tele-Tutor.

- Wenn jemand in einem Online-Seminar Aufgaben in virtuellen Kleingruppen bearbeitet oder Mitglied einer virtuellen Interessengemeinschaft ist, in der Gleichgesinnte ihre Erfahrungen zu bestimmten Problemen austauschen, kann man ebenfalls von E-Learning sprechen. Die Funktion der neuen Medien bei dieser Form des E-Learning besteht darin, Lernende an verschiedenen Orten miteinander in Kontakt zu bringen und sie zu einer gemeinsamen Problemlösung im virtuellen Raum anzuregen. Man könnte daher von einem E-Learning by collaborating sprechen; die neuen Medien übernehmen hier die Funktion, eine Kollaboration ${ }^{1}$ zwischen Lernenden anzustossen.

Aus der Sicht des Lerners besteht diese Form des E-Learning darin, relativ eigenständig neues Wissen in der Lernumgebung zu konstruieren und dies vor allem im Prozess des sozialen Problemlösens zu tun. Beim E-Learning by collaborating ist ein Lehrender als Initiator und Moderator von Lernprozessen oder als Coach beim virtuellen Problemlösen unabdingbar.

\subsection{Verschiedene Anforderungen beim E-Learning}

E-Learning by distributing, E-Learning by interacting und E-Learning by collaborating sind drei Varianten von Lernen mit neuen Medien, die unterschiedliche Anforderungen stellen, und zwar zum einen an die Gestaltung der E-Learning-Umgebung und damit an die Fähigkeiten der Mediengestalter und der Lehrenden und zum anderen an die Lernprozesse und damit an die Voraussetzungen, die die Lernenden mitbringen müssen. Bei allem Eifer für das E-Learning werden beide Aspekte in der Praxis gerne vernachlässigt.

\footnotetext{
Im Deutschen ist der Begriff der Kollaboration wenig üblich, meist wird er mit «Kooperation» gleichgesetzt. Was Kollaboration von Kooperation vor allem in der englischsprachigen Literatur unterscheidet, ist die Akzentuierung der Wissensteilung und der gemeinsamen Wissenskonstruktion.
} 
- Wenn die neuen Medien zur Distribution von Informationen eingesetzt werden, stellen sich Lernprozesse nur ein, wenn die Gestaltung der Information auch lernfreundlich ist, wenn multimediale Potentiale ausgeschöpft, aber auch zielsicher eingesetzt werden. Kurz: Wenn das Design von Text und Bild ebenso stimmt wie die Struktur und Aufbereitung der Information (z. B. Weidenmann, 2002) - was bekanntlich im weltweiten Netz bei Leibe nicht immer der Fall ist.

Die Anforderungen an den Lernenden bei dieser Form des E-Learning sind hoch: Denn E-Learning by distributing funktioniert - etwas böse formuliert - nach dem Prinzip «Friss oder stirb»: Die Information zum Lernen ist da, aber der Antrieb, diese auch zu lesen, zu verstehen und zu nutzen muss aus einem selbst heraus kommen, was Motivation und meist auch Vorwissen voraussetzt. Ebenso sind Fähigkeiten zur Selbstbestimmung und Selbststeuerung (Friedrich \& Mandl, 1997) sowie zum Umgang mit den neuen Medien, also Medienkompetenz, eine Bedingung dafür, dass Lernen auf diesem Wege überhaupt stattfindet.

- Wenn die neuen Medien zur Interaktion zwischen Nutzer und System dienen, muss zur lernfreundlichen Informationsgestaltung auch eine professionelle Gestaltung von Instruktionen, Übungen, Aufgaben und Rückmeldungen kommen (z. B. Leutner, 2002). Auch hier hinkt die Wirklichkeit dem Anspruch oft hinterher, was Benutzer von CBTs und WBTs sicher bestätigen können.

Die Anforderungen an den Lernenden bei dieser Form des E-Learning sind eher niedrig: Denn E-Learning by interacting funktioniert - wieder etwas provokativ formuliert - nach dem Prinzip «Mit Zuckerbrot und Peitsche»: Nicht nur die aufbereitete Information zum Lernen ist da, auch der notwendige «Trainingsapparat» ist bereit, um zu loben und zu piesacken, bis Wissen und Fertigkeiten endlich sitzen. Freilich setzt auch das beim Lernenden ein ausreichendes Mass an Motivation und Fähigkeiten zur Selbstorganisation am «Trainingsgerät» voraus.

- Wenn die neuen Medien zur Unterstützung der Kollaboration zwischen Lernenden eingesetzt werden, müssen Lernumgebungen neben didaktisch überlegter Gestaltung von Information, Instruktion und Aufgaben auch geeignete inhaltliche und soziale Kontexte bereitstellen. Das heisst: Kooperative Lernprozesse im virtuellen Raum stellen sich nur ein, wenn Problemstellungen so gewählt sind, dass sich eine Zusammenarbeit für die Lernenden auch lohnt; zudem muss die soziale Situation die Zusammenarbeit wirksam unterstützten (Reinmann-Rothmeier \& Mandl, 2002). Das hierzu erforderliche didaktische Know-how wird chronisch unterschätzt.

Die Anforderungen an den Lernenden bei dieser Form des E-Learning sind sehr hoch: E-Learning by collaborating gilt zwar immer häufiger als Königsweg des Lernens mit neuen Medien - man denke nur an die ganze Community-Bewegung (z. B. Bielaczyc \& Collins, 1999): Doch Kooperation ist generell schwierig und verlangt unter virtuellen Bedingungen ein besonders hohes Mass an sozialer Kompetenz, an Selbststeuerungsfähigkeit und Medienerfahrung. Lernen durch virtuelles Problemlösen in der Gruppe hat manchmal was vom Prinzip «Mitgefangen - mitgehangen»: Denn auch in virtuellen Teams gibt es sozialen Druck; die Gefahr des Scheiterns durch Trittbrettfahrer ist gross; und die fehlende soziale Unmittelbarkeit bringt neue Probleme mit sich. 
Die nachfolgende Tabelle stellt die verschiedenen Aussagen zu den drei ELearning-Varianten noch einmal zusammen.

Tab. 1: Drei E-Learning-Varianten

\begin{tabular}{|l|l|l|l|l|}
\hline $\begin{array}{l}\text { Leitfunktion } \\
\text { Medien zur }\end{array}$ & $\begin{array}{l}\text { E Learning } \\
\text { durch: }\end{array}$ & $\begin{array}{l}\text { Anforderungen an den } \\
\text { Lernenden }\end{array}$ & $\begin{array}{l}\text { Aufgaben des } \\
\text { Entwicklers / } \\
\text { Mediengestalters }\end{array}$ & $\begin{array}{l}\text { Rolle des } \\
\text { Lehrenden }\end{array}$ \\
\hline $\begin{array}{l}\text { Distribution } \\
\text { von } \\
\text { Information }\end{array}$ & $\begin{array}{l}\text { Informations- } \\
\text { rezeption + } \\
\text { selbstgesteuerte } \\
\text { Informations- } \\
\text { verarbeitung }\end{array}$ & $\begin{array}{l}\text { Selbststeuerungs- } \\
\text { fähigkeit; } \\
\text { Medienkompetenz; } \\
\text { ausreichendes } \\
\text { Vorwissen; insg. hohe } \\
\text { Anforderungen }\end{array}$ & $\begin{array}{l}\text { Lernfreundliche } \\
\text { Informations- } \\
\text { gestaltung }\end{array}$ & $\begin{array}{l}\text { Keine Personen } \\
\text { in der Rolle des } \\
\text { Lehrenden er- } \\
\text { forderlich }\end{array}$ \\
\hline $\begin{array}{l}\text { Interaktion } \\
\text { zw. Nutzer + } \\
\text { System }\end{array}$ & $\begin{array}{l}\text { angeleitete } \\
\text { Informations- } \\
\text { verarbeitung + } \\
\text { selbstorgani- } \\
\text { siertes Üben }\end{array}$ & $\begin{array}{l}\text { Motivation; Fähigkeit } \\
\text { zur Selbstorganisation } \\
\text { insg. } \text { eher niedrige } \\
\text { Anforderungen }\end{array}$ & $\begin{array}{l}\text { Infogestaltung + } \\
\text { Gestaltung von } \\
\text { Instruktionen, } \\
\text { Übungen, Auf- } \\
\text { gaben sowie Feed- } \\
\text { back + Antworten }\end{array}$ & $\begin{array}{l}\text { Lernberater } \\
\text { oder Tele- } \\
\text { Tutor möglich }\end{array}$ \\
\hline $\begin{array}{l}\text { Kollaboration } \\
\text { zw. Lernenden }\end{array}$ & $\begin{array}{l}\text { Eigenständige } \\
\text { Wissenskon- } \\
\text { struktion + } \\
\text { soziales } \\
\text { Problemlösen }\end{array}$ & $\begin{array}{l}\text { Selbststeuerungs- } \\
\text { fähigkeit; Medien- } \\
\text { erfahrung; soziale } \\
\text { Fähigkeiten; } \\
\text { insg. sehr hohe } \\
\text { Anforderungen }\end{array}$ & $\begin{array}{l}\text { Lernfreundliche } \\
\text { Infogestaltung+ } \\
\text { Gestaltung von } \\
\text { Instruktionen, } \\
\text { Aufgaben sowie } \\
\text { inhaltlichen + } \\
\text { sozialen } \\
\text { Kontexten }\end{array}$ & $\begin{array}{l}\text { Lehrender als } \\
\text { Initiator und } \\
\text { Moderator/ } \\
\text { Coach notwendig }\end{array}$ \\
\hline
\end{tabular}

\section{Pädagogisch-psychologisches Modell zum Wissensmanagement}

3.1 Der Managementbegriff

Der Begriff des Managements ist im Rahmen der Pädagogik eher unüblich und entsprechend schwer vermittelbar (Reinmann-Rothmeier \& Mandl, 2000). Das Wort «Managen» löst unter Pädagogen vor allem Assoziationen wie Lenken, Steuern und Kontrollieren z. B. von personellen und materiellen Ressourcen aus. Da Management im Sinne von Geschäftsführung mit genau diesen Dingen zu tun hat, ist diese Vorstellung nicht falsch, aber sie ist unvollständig: Denn Geschäftsführung ist nur eine Seite der Management-Medaille; die andere heisst Mitarbeiterführung und dabei geht es darum, Lern- und Entwicklungsprozesse von Mitarbeitern zu initiieren und zu begleiten, Arbeits- und Lernumgebungen zu gestalten, Feedback zu geben, zu coachen und zu beraten.

Dieses Managementverständnis nimmt allerdings auch wieder nur eine von mindestens zwei möglichen Perspektiven ein, nämlich die Perspektive der Organisation. Schlagworte wie «Zeitmanagement», «Konfliktmanagement» oder «Selbstmanagement» verweisen darauf, dass der Managementbegriff immer häufiger auch Anwendung auf einer rein psychologischen Ebene findet, auf der Individuen und kleinere Gruppen, nicht aber die Organisation als Ganzes im Vordergrund stehen. Und diese zweite Perspektive spielt auch beim Wissensmanagement aus einer pädagogisch-psychologischen Perspektive eine ganz zentrale, aber oft vernachlässigte Rolle (ReinmannRothmeier \& Mandl, 2001).

Der zweite Teil des Wissensmanagementbegriffs - also das Wissen - ist freilich ein noch komplexerer Begriff, den man aus erkenntnistheoretischem Interesse ebenso beleuchten kann wie vor dem Hintergrund psychologischer, pädagogischer und auch organisationstheoretischer Fragestellungen. Es würde den Rahmen sprengen, an dieser Stelle den Wissensbegriff im Detail zu beleuchten (vgl. Eckert, 1998)

Wenn im Folgenden ein heuristisches Modell zum Wissensmanagement aus pädagogisch-psychologischer Sicht skizziert wird, soll auf den Wissensbegriff soweit es zum Verständnis notwendig ist, noch eingegangen werden (vgl. Reinmann-Rothmeier, Mandl, Erlach \& Neubauer, 2001).

3.2 Vier Prozessbereiche im Umgang mit Wissen

Eine zentrale Frage des Wissensmanagements bestand von Anfang an darin, wie man Wissen am besten «festhält» und greifbar macht, wie man es ablegt, aufbewahrt und wiederfindet. Viele Wissensinhalte lassen sich heute elektronisch speichern; Datenbanken sollen Wissen nach verschiedenen Strukturen transparent, jederzeit zugänglich und nutzbar machen. Dabei kann man z. B. sog. Wissenslandkarten einsetzen, um Orte, Formen und Mengen an Wissen, die es in einer Organisation gibt, zu kartografieren. Dabei geht es stets um die Repräsentation von Wissen, bei der die 
Medien eine wichtige (aber nicht die einzige) Rolle spielen. Viele Organisationen haben sich dieser ersten Welle des Wissensmanagements angeschlossen, möglichst viel Information und Wissen dokumentiert und damit eine Art «Wissensmanagement by Eichhörnchen» (Schneider, 2001) betrieben. Nach den ersten Wissensmanagement-Erfahrungen ist deutlicher geworden, dass man sich bei der Wissensrepräsentation nicht auf technische Systeme allein verlassen sollte. Insbesondere implizite Wissensanteile (Polanyi, 1966) entziehen sich all den Methoden, die eine verbale Artikulation von Wissen voraussetzen.

Ein weniger bekanntes, aber praktisch sehr bedeutsames Wissensmanagement-Problem besteht darin, dass Organisationen zwar über Wissen verfügen, dieses Wissen aber oft nicht zur Anwendung kommt. Hier haben wir es mit einem ähnlichen Phänomen wie dem «trägen Wissen» zu tun, das Pädagogen aus Lehr-Lernsituationen nur allzu gut kennen (Renkl, 1996). In diesen Zusammenhang gehört auch das sog. «Not-invented-hereSyndrom», das den Zustand beschreibt, nur ja nichts von anderen zu übernehmen, weil dies als Schwäche oder als Unfähigkeit ausgelegt werden könnte, selbst etwas auf die Beine zu stellen. Man kann dies als Nutzung von Wissen bezeichnen - ein Prozessbereich, den man im Wissensmanagement lange Zeit übersehen hat, wahrscheinlich deshalb, weil es dafür keine technischen Tools gibt. Denn handeln müssen nach wie vor die Menschen in einer Organisation - genau dies können ihnen technische Systeme nicht abnehmen. Wissensnutzung meint die Umsetzung von Wissen in Entscheidungen und Aktivitäten, also wissensbasiertes Handeln. Sog. Best PracticeBerichte und Lessons Learned sind klassische Wissensmanagement-Massnahmen, die helfen sollen, vorhandenes Wissen noch einmal zu nutzen und Fehler nicht ein zweites und drittes Mal zu wiederholen (vgl. Probst, et al., 2000). Auch hier aber stehen Wissensmanager nach wie vor vor Problemen, wenn es um die Anwendung impliziten Wissens geht, wie es beispielsweise bei langjährig aufgebautem Erfahrungswissen der Fall ist. Pädagogisch-psychologisches Know-how hat also auch hier seinen Platz.

Ein weiteres Ziel, das nun schon seit mehreren Jahren die Wissensmanagement-Szene dominiert, lautet «Knowledge Sharing». Auch in Bezug auf das Knowledge-Sharing setzte man - ähnlich wie bei der Wissensrepräsentation - anfangs vor allem auf Technik, was erst einmal nahe liegend scheint: Nie zuvor hat man in Organisationen auf so viele technische Kommunikationsmöglichkeiten zurückgreifen können wie heute: Vom guten alten Brief über das «normale» und das Bild-Telefon bis zu E-Mail, Computer- und Videokonferenzen, asynchronen Diskussionsoren und elektronischen Chats in Echtzeit. Insbesondere sog. Communities of Practice, die meist virtuell arbeiten, haben sich in grösseren Unternehmen einen festen Platz für die Weitergabe von Wissen erkämpft (Romhardt, 2002). Letztlich aber funktioniert auch das beste Knowledge-Sharing System nicht, wenn die Beteiligten dieses nicht akzeptieren, wenn sie nicht motiviert sind, sich daran wirklich zu beteiligen. Die Kommunikation von Wissen in einer Organisation, wie man diesen Bereich an Wissensprozessen auch nennen kann, setzt in jedem Fall mehr als die Einführung eines Intranets voraus. Zur Wissenskommunikation zählt letztlich sowohl die Weitergabe kontextunabhängigen Wissens als auch der Austausch und das Teilen von Erfahrung zwischen den Mitgliedern einer Organisation.

Die Repräsentation, Nutzung und Kommunikation von Wissen sind Prozesse, die bereits bestehendes Wissen voraussetzen. Da man in Organisationen nicht mit ausschliesslich «altem» Wissen auskommen kann, muss man im Wissensmanagement auch Massnahmen ergreifen, um an neue Informationen zu kommen, neues Wissen aufzubauen und innovative Ideen hervorzubringen. Die Generierung von Wissen ist ein Bereich des Wissensmanagements, der nahe an Fragen der Innovation heranrückt. Angesichts komplexer Probleme und Aufgaben in heutigen Organisationen ist die Generierung neuen Wissens selten etwas, das man im Alleingang macht: Wissensgenerierung ist meist ein sozialer Prozess; er setzt den Austausch von Wissen und Erfahrung ebenso voraus wie das aktive Tun. Auch bei der Wissensgenerierung kann man mit technischen Tools eher wenig ausrichten, auch wenn beispielsweise Visualisierungsverfahren durchaus helfen können, kreative Prozesse zu unterstützen. Entscheidend aber ist auch bei diesem Bereich von Wissensprozessen, dass sich die dahinter stehende individuelle Aktivität für den Einzelnen lohnt, dass die Menschen in einer Organisation motiviert und natürlich auch fähig sind, neues Wissen aufzubauen und kontinuierlich zu lernen.

Das vorliegende Modell zum Managen von Wissen (das Münchener Modell, vgl. Reinmann-Rothmeier, 2001) bündelt die vielen möglichen Prozesse im Umgang mit Wissen zu vier Prozessbereichen, die gerade mit einigen Beispielen skizziert wurden:

- Prozesse der Repräsentation von Wissen bringen Wissen in ein sichtbares oder hörbares Format, machen es transparent und greifbar, indem 
es z. B. aufgeschrieben, aufgemalt, digitalisiert u. ä. wird. Wichtig ist dabei, dass es nicht nur verbale Möglichkeiten der Dokumentation von Wissen gibt, sondern auch bildhafte oder analoge Repräsentationsformen. Wissen nähert sich mit diesen Prozessen der Information, die man auch unabhängig von Personen und Kontexten aufbewahren, zur Verfügung stellen und verteilen kann.

Um sich diesen Bereich des Wissensmanagements besser einzuprägen und anschaulicher zu machen, kann man die Wissensrepräsentation mit dem Einfrieren von Wasser vergleichen: Man macht Wasser zu Eis, das man anfassen und aufbewahren, weitergeben, konservieren, stapeln, aber auch wieder auftauen kann.

- Während sich Wissen bei Repräsentationsprozessen vom Zustand her der Information nähert, rückt das Wissen bei seiner Nutzung nah an das Handeln heran. Die Nutzung von Wissen meint Prozesse, mit denen man Wissen in Entscheidungen, Massnahmen und Aktivitäten zur Anwendung bringt, es sozusagen lebendig macht und ein träges Verbleiben von Information und Wissen in Köpfen, auf Papier oder elektronischen Trägern verhindert.

Vergleichen kann man die Wissensnutzung mit dem Verdampfen von Wasser: Man macht Wasser zu Wasserdampf, der zwar flüchtig und nicht mehr unmittelbar greifbar ist, aber hohe Mengen wertvoller Energie erzeugen und letztlich auch wieder kondensieren kann.

- Unabhängig davon, ob man es eher mit informationsnahem oder handlungsnahem Wissen zu tun hat: Wissen kann man in jedem Zustand in Bewegung bringen, indem man es verteilt oder mit anderen teilt. Gängig ist auch die Bezeichnung «Wissensfluss»; und in der Tat liegt gerade bei der Kommunikation von Wissen der Vergleich zum Wasser besonders nahe.

Wissen ist wie Wasser etwas Fliessendes: Es kann sich bewegen und ausbreiten, sich dabei aber auch verändern - man denke an den Spruch, dass man nicht zweimal in denselben Fluss steigen kann.

- Wissen transparent machen, es zur Anwendung und in Bewegung bringen - das sind Prozesse, die mit bestehendem Wissen arbeiten. Von daher müssen im Wissensmanagement auch Vorgänge hinzukommen, durch die neues Wissen entstehen kann. Die Generierung von Wissen meint Prozesse, mit denen neue Ideen hervorgebracht und neue Erfahrungen und Erkenntnisse gemacht werden.

Vergleichbar ist die Wissensgenerierung mit der Quelle des Wassers, die dafür sorgt, dass der Fluss nicht zum Erliegen kommt.

Diese vier Prozessbereiche sind nicht trennscharf und auch nicht voneinander unabhängig. Das Münchener Modell will keine theoretischen Erkenntnisse und auch keine Rezepte an die Hand geben. Vielmehr handelt es sich um ein heuristisches Modell, das einem hilft, die geschilderten Wissensprozesse in Gang zu setzen und zu unterstützen.

\subsection{Lernende Organisation als Ziel}

- Wissensmanagement im hier gemeinten Sinne steht und fällt mit der Verzahnung der vier Prozessbereiche sowie mit der Verzahnung psychologischer und organisationaler Vorgänge und Bemühungen. Im Münchener Modell wird daher ein enger Bezug zum organisationstheoretischen Modell von Senge et al. (Senge, Kleiner, Smith, Roberts \& Ross, 1997) hergestellt, demzufolge erst die Verbindung individueller und organisationaler Lernprozesse den Boden dafür ebnet, dass sich Organisationen zu lernenden Organisationen entwickeln. Individuelles und organisationales Lernen - das ist auch das Ziel von Wissensmanagement aus einer pädagogisch-psychologischen Sicht. Dabei gilt nach Senge et al., dass der Mensch der eigentliche Ort des Wandels, die Organisation vor allem Ort des Handelns ist. Was heisst das genau?

- Den Menschen als Ort des Wandels zu begreifen bedeutet, dass man das individuelle Lernen in den Vordergrund stellt und folgenden Dreierschritt postuliert: Menschen brauchen zunächst Wissen und Können, um Möglichkeiten für Veränderungen in ihrer Umgebung überhaupt wahrnehmen zu können; eine bewusste Wahrnehmung und Sensibilität wiederum ist die Voraussetzung dafür, dass sich auch Einstellungen und Überzeugungen wandeln.

- Die Organisation als Ort des Handelns zu verstehen, hat zur Folge, dass auch das organisationale Lernen von Interesse ist; auch hier postulieren Senge et al. (1997) einen Dreierschritt: Die Organisation braucht zunächst einmal eine Leitidee, die Orientierung gibt und Begeisterung 
schafft; dazu kommen müssen Konzepte und Methoden, mit denen Leitideen realisierbar werden; schliesslich bedarf es bestimmter Strukturen in der Organisation, die die Umsetzung von Konzepten und Methoden fördern und nicht etwa torpedieren.

Mit den beiden Lernzyklen von Senge et al. als Ziel und den vier Prozessbereichen als Orientierung für mögliche Wissensprozesse spannt das Münchener Modell für das Wissensmanagement zwei Dimensionen auf: (a) die Inhaltsdimension, die die Frage beantwortet: Was kann oder soll man mit Wissen in einer Organisation tun - das sind die vier Prozesskategorien; (b) die Trägerdimension, die die Frage beantwortet: Wo können oder sollen Wissensmanagementprozesse initiiert und praktiziert werden - das sind die beiden Lernorte oder Lernzyklen.

Die folgende Abbildung 2 fasst die wichtigsten Aussagen um das Münchener Wissensmanagement-Modell noch einmal zusammen:

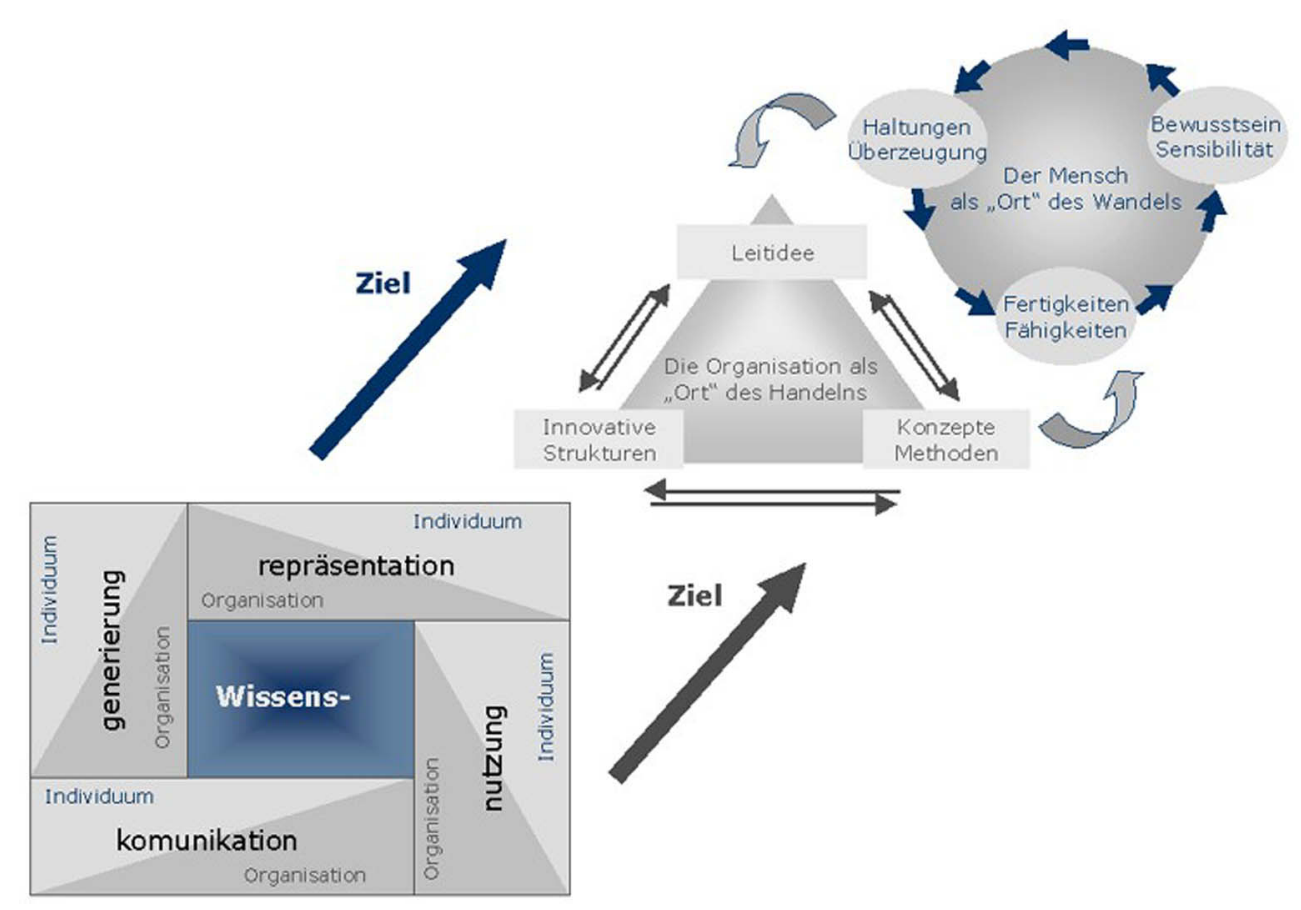

Abb. 2: Ein pädagogisch-psychologisches Wissensmanagement-Modell $17 / 27$

\section{Mediendidaktik und Wissensmanagement - Potentiale für eine gegenseitige Bereicherung}

Wie bereits erwähnt wurde, kommt es vor allem durch den E-Learning Trend in der Wirtschaft sowie durch das langsam aufkommende Interesse am Thema Wissensmanagement in (medien-) pädagogischen Bereichen zu ersten Annäherungen zwischen Mediendidaktik und Wissensmanagement (vgl. Abschnitt 1.3). Aus der Sicht der Wirtschaft sind es vor allem technologisch motivierte Gründe, die dafür sprechen, E-Learning und Wissensmanagement miteinander zu verschmelzen. Anhand der hier skizzierten Modelle zu E-Learning und Wissensmanagement aus einer pädagogisch-psychologischen Sicht sprechen freilich andere Gründe für dieselbe Entwicklungsrichtung.

\subsection{Verbindung von Wissensmanagement und E-Learning}

aus wirtschaftlicher Perspektive

Innerhalb der Wirtschaft mehren sich die Anzeichen dafür, E-Learning mit Wissensmanagement (und umgekehrt) miteinander in Beziehung zu setzen und zu verbinden. Zwar gibt es nach wie vor Differenzen nicht nur in den Begriffswelten, sondern auch in Bezug auf strategische, organisationale und politisch-kulturelle Kriterien von E-Learning und Wissensmanagement in wirtschaftlichen Organisationen (Back, 2002, Kap. 7.1., S. 5f.): So ist etwa Wissensmanagement im Vergleich zu E-Learning strategisch und organisatorisch enger an bestimmte Geschäftsbereiche und zentrale Stellen gekoppelt; auf der inhaltlichen Ebene herrschen im Wissensmanagement (noch) eher kurzlebige, wenig bis gar nicht aufbereitete Informationen vor, während man sich beim E-Learning auf langlebige und didaktisch strukturierte Themen konzentriert. Trotz dieser und anderer Unterschiede lässt sich eine Tendenz zu integrierten Lösungen beobachten, mit denen zumindest die technischen Grundlagen von Wissensmanagement und ELearning zusammengeführt werden. Auch werden immer häufiger ELearning-Tools zum Wissensmanagement und umgekehrt genutzt. Sehr häufig ist dies der Fall bei Instrumenten aus dem $\mathrm{CSCW}^{2}$-Bereich: Solche Tools zur virtuellen Kooperation lassen sich zum elektronischen Wissenstransfer beim Wissensmanagement ebenso heranziehen wie beim kooperativen Lernen in virtuellen Gruppen; in diesem Zusammenhang spricht man neuerdings auch von «E-Collaboration» (Back, 2002). Ein weiteres Bei-

\footnotetext{
${ }^{2}$ Computer Supported Cooperative Work
} 
spiel sind technische Tools, die speziell für das «learning-just-in-time», also für kurze Lernsequenzen im Arbeitsprozess entwickelt werden: Hier handelt es sich einerseits um eine typische Ausprägung des E-Learning, andererseits aber auch um eine Möglichkeit, den Zugang zu notwendigem Wissen und/oder den raschen Aufbau neuen Wissens für die Lösung konkreter Probleme im Arbeitsprozess zu ermöglichen (z. B. Back et al., 2001).

«Es ist zu erwarten, dass E-Learning, Electronic-Human-Resource-Management (E-HRM) ${ }^{3}$, E-Business \& M-Commerce ${ }^{4}$ sowie Wissensmanagement konvergieren. Etwas metaphorisch könnte man von einer neuen Generation des E-Learning sprechen. Die jeweils im Hintergrund stehenden Informationstechniken, d. h. Lernplattformen, People-Portals ${ }^{5}$, KnowledgeTools sowie E-Business \& Ubiquitous-Computing-Devices ${ }^{6}$, verschmelzen» (Wilbers, 2002, Kap. 4.0, S. 12). So oder ähnlich wird derzeit von ökonomischer Seite die Zukunft des Wissens und Lernens mit neuen Medien formuliert. Angesichts der damit verbundenen Konvergenz von geschäftsbezogenen Wissensprozessen und Prozessen des individuellen und organisationalen Lernens kommt der Gestaltung medialer Lern- und Arbeitsumgebungen eine nicht zu unterschätzende Rolle zu; entsprechend wichtig und dringend sollte das dazu erforderliche mediendidaktische Know-how sein. Es dürfte allerdings nicht verwundern, dass Mediendidaktiker zurückhaltend bis ablehnend reagieren, wenn ihnen Zukunftsbeschreibungen wie die obige zu Ohren kommen. Das liegt nicht nur an den Begrifflichkeiten, die sich auch mit einfachen Worten erklären lassen; es liegt vor allem daran, dass man den technischen Entwicklungen wieder einmal Priorität vor (pädagogisch motivierten) Fragen einräumt, die vom Lernenden, vom Wissensträger und von den Zielen einer lernenden Organisation ausgehen.

Im Folgenden soll versucht werden, aufbauend auf dem Orientierungsmodell zum E-Learning (siehe Abschnitt 2) und dem pädagogisch-psycho-

${ }^{3}$ Z. B. internetgestützte Personalauswahl oder elektronisch gestützte Einarbeitung neuer Mitarbeiter

${ }^{4}$ Handel mit mobilen Kommunikationsgeräten (z. B. Handys)

5 Personalisierter Zugang zu spezifischen Themen/Inhalten im Internet über eine Plattform

${ }^{6}$ Ubiquitous Computing meint die Integration neuer Kommunikationstechnologie in den Alltag, ohne dass man es «merkt», was mit Hilfe drahtloser Übertragung und Miniaturisierung immer besser möglich wird

$19 / 27$ logischen (Münchener) Modell zum Wissensmanagement (siehe Abschnitt 3) eine andere Form der Verbindung von Mediendidaktik (respektive ELearning) und Wissensmanagement herauszuarbeiten.

4.2 Verbindung von Wissensmanagement und E-Learning aus pädagogischpsychologischer Perspektive

Aus pädagogisch-psychologischer Perspektive geht die Suche nach Verbindungen zwischen E-Learning und Wissensmanagement nicht von technischen Argumenten aus, wie sie kurz beschrieben wurden (vgl. Abschnitt 4.1). Vielmehr stehen die psychologischen Prozesse im Vordergrund, die beim Umgang mit Wissen sowie beim Lernen mit neuen Medien ablaufen. Diese nämlich bilden die Basis für die Entwicklung und Umsetzung mediendidaktischer Massnahmen zur Förderung von Wissen und Lernen in medialen Umgebungen. Zu diesem Zweck wird im Folgenden auf die vier Prozessbereiche des Wissensmanagements aus dem Münchener Modell (Repräsentation, Nutzung, Kommunikation und Generierung von Wissen) zurückgegriffen sowie auf die in Anlehnung an Back et al. (1998) formulierten E-Learning-Varianten (E-Learning by distributing, by interacting und by collaborating).

Organisationen, die Informationen nutzergerecht und übersichtlich aufbereiten und technisch gestützt, aber auch gezielt verteilen, erhöhen die Wahrscheinlichkeit, dass ihre Mitglieder im Zuge von Informationsrecherchen im Netz auch lernen. Oder anders formuliert: Geschickte Repräsentation und Kommunikation von Wissen können den nützlichen «Nebeneffekt» eines E-Learning by distributing nach sich ziehen. Diese Verbindungsmöglichkeit ist nicht nur für wirtschaftliche Organisationen interessant: Auch Bildungsinstitutionen können neue Möglichkeiten für ein E-Learning by distributing schaffen, wenn sie vorhandene Informationen (etwa Lehr-Lernmaterialien in der Schule, Infos über Forschungsförderungen an der Hochschule etc.) elektronisch zugänglich machen, professionell gestalten und technisch versiert verbreiten. «Professionell gestalten» heisst, mediendidaktische Erkenntnisse heranzuziehen, wenn es darum geht, bei der Auswahl, Aufbereitung und Darstellung von Information und Wissen Gefahren wie kognitive Überlastung zu reduzieren, das Zusammenspiel verschiedener Symbolsysteme zu berücksichtigen und dabei auch noch Motivation und Interesse zu fördern - um nur ein paar Beispiele zu nennen (z. B. Weidenmann, 2002; Schnotz, 2002). 
Prozesse der Repräsentation und Kommunikation von Wissen können in Organisationen an verschiedene Formen des E-Learning by distributing gekoppelt werden und umgekehrt. Eine nutzerfreundliche Aufbereitung von Inhalten kann dazu beitragen, sowohl Wissensmanagement- als auch Lernprozesse zu fördern.

Organisationen, die den Austausch, die Weitergabe und das Teilen von Wissen als sozialen Lernprozess verstehen, können die Wissenskommunikation im Wissensmanagement hervorragend mit E-Learning by collaborating kombinieren: Denn soziales Problemlösen in virtuellen Gruppen, das pädagogisch-didaktisch unterstützt und begleitet wird, kann eher Einstellungen, Erkenntnisse und Fähigkeiten zum Teilen von Wissen fördern als Massnahmen, die nur auf Technik oder materielle Anreize setzen. Zugleich besteht die Chance, dass durch Kooperation auch neues Wissen entsteht auf das einer allein nicht gekommen wäre. Das heisst: E-Learning by collaborating kann den Wissensaustausch ebenso wie die soziale Wissensgenerierung wirksam unterstützen. Auch diese Verbindung ist keineswegs wirtschaftlichen Organisationen vorbehalten: Schulen und Hochschulen sowie andere soziale Organisationen können mit einer Aufwertung des kooperativen Lernens ebenfalls den Wissensfluss sowie einen anwendungsbezogenen (situativen) Aufbau neuen Wissens fördern: Beispielhaft zu nennen wären virtuelle Learning Communities in der Jugendarbeit, in denen Wissen und Erfahrung zur Medienkompetenz Jugendlicher ausgetauscht und in gemeinsamen Projekten nach praktisch umsetzbaren Ideen gesucht werden (Bader, 2001). Praxiserfahrungen und empirische Befunde legen es jedoch nahe, Lern- und Wissensprozesse in Gruppen nicht dem Zufall zu überlassen. Mediendidaktische Massnahmen sind an vielen Stellen notwendig: Es müssen geeignete Aufgaben sowie verständliche Anleitungen und Instruktionen entwickelt, kooperative Prozesse technisch und mit Hilfe von Tutoren oder Moderatoren unterstützt und geeignete Materialien für die Zusammenarbeit bereit gestellt werden, damit die Kooperation in der (virtuellen) Gruppe die erhofften Wissens- und Lernprozesse tatsächlich zur Folge hat (vgl. Reinmann-Rothmeier \& Mandl, 2002).

Prozesse der Kommunikation und Generierung von Wissen lassen sich in Organisationen mit E-Learning by collaborating verbinden. Eine didaktisch sinnvolle Gestaltung und Unterstützung kooperativen Lernens und Arbeitens mit neuen Medien verbessert nicht nur den Wissensaustausch, sondern erhöht auch die Chance eines individuellen und kollektiven Wissenszuwachses.
Werden Wissensprozesse - wie im Münchener Modell - sowohl auf der organisationalen als auch auf der individuellen Ebene verortet, lassen sich alle Varianten des E-Learning (auch E-Learning by interacting) beim Managen von Wissen nutzbar machen. Wer die individuelle Wissensgenerierung der Organisationsmitglieder mit Hilfe der neuen Medien fördert, tut auch der Organisation als Ganzes etwas Gutes - erinnert sei an Senges individuellen Lernzyklus und daran, dass letztlich nur der Mensch der eigentliche Ort des Wandels ist (vgl. Abschnitt 3.3). Von daher ist ein Wissensmanagement in Organisationen ohne Förderung individuellen Lernens gar nicht möglich - entsprechend gezielt kann man freilich auch ELearning-Varianten zu eben dieser individuellen Lernförderung einsetzen. Eine weitere Unterstützung erfährt diese Form der Verbindung von ELearning und Wissensmanagement durch die Erkenntnis, dass Arbeitsumgebungen auch Lernumgebungen sind (z. B. Schlaffke, 1999): Lernen am Arbeitsplatz gilt als besonders effizient und vor allem auch anwendungsorientiert, sodass die typische Gefahr des «trägen Wissens» etwa nach Seminarbesuchen reduziert wird. Für Lernprozesse «on demand» im Arbeitsprozess bieten sich sowohl E-Learning by distributing (z. B. Recherchen in Datenbanken oder Expertenverzeichnissen) als auch E-Learning by interacting (z. B. kurze Web Based Trainings für den raschen Erwerb umgrenzter Fertigkeiten) an. Erfolgreich aber sind diese E-LearningVarianten nur dann, wenn sie mediendidaktisch gestützt, also lernfreundlich gestaltet sind und den Lernenden zu einer aktiven Wissenskonstruktion anregen - was bis dato häufig nicht der Fall ist! Diese Verbindung von ELearning und Wissensmanagement klingt zunächst nur für wirtschaftliche Organisationen interessant. Aber auch Schulen könnten davon profitieren: z. B. im Rahmen der sog. 20-Minuten-Fortbildung77, bei der Experten für bestimmte Themen innerhalb der Schule identifiziert werden, die dann eine kurze interne Form anbieten und als Ansprechpartner zur Verfügung stehen - was sich auch medial unterstützen liesse.

Prozesse der Generierung und Nutzung von Wissen können mit allen ELearning-Varianten verknüpft werden. Insbesondere die für das Wissensmanagement notwendigen individuellen Lernprozesse machen E-LearningVarianten notwendig, die pädagogisch-didaktische Erkenntnisse zum Lernen mit neuen Medien berücksichtigen und umsetzen.

\footnotetext{
${ }^{7}$ siehe: http://www.netzwerk-medienschulen.de
} 
Im wirtschaftlichen Kontext wird vermehrt betont, dass eine Verbindung von Wissensmanagement und E-Learning dazu beitragen kann, die Entwicklung von individuellen Kenntnissen und Kompetenzen (als eine genuine Aufgabe der Personalentwicklung) stärker an die Unternehmensziele zu binden (Back, 2002). Damit kann und soll eine Aufwertung von individuellem Wissen und Lernen auf der strategischen Ebene einhergehen. Auch für Bildungsinstitutionen kann diese Argumentation von Bedeutung sein: Im Rahmen der Schulentwicklung etwa wird zunehmend erkannt, dass erst das Ineinandergreifen von Organisations-, Personal- und Unterrichtsentwicklung die geforderte Schulentwicklung bewirken kann, von der man erwartet, dass sie die Schule zu einer lernenden Organisation macht (Kempfert \& Rolff, 1999). Das Lernen mit neuen Medien wiederum kann sowohl der Unterrichts- als auch der Personalentwicklung wichtige Impulse geben; auch Ideen aus dem Bereich des Wissensmanagements werden bereits erfolgreich in Schulen zur Organisationsentwicklung eingesetzt ${ }^{8}$. Von daher lohnt sich der Versuch auch für Bildungsinstitutionen, dem individuellen und organisationalen Lernen durch Wissensmanagement einen neuen, strategisch höheren Stellenwert beizumessen.

Wissensmanagement kann als Impuls dafür fungieren, das Lernen im Allgemeinen und das E-Learning im Besonderen stärker an die strategischen Ziele einer Organisation anzubinden und den Stellenwert des Lernens für die Weiterentwicklung einer Organisation zu erhöhen.

\section{Mediendidaktik und Wissensmanagement -}

ein Paar mit komplementären Potentialen

Die Industrie gibt sich an pädagogisch-psychologischen Fachbereichen nicht gerade die Klinke in die Hand: Und so muss es nicht verwundern, dass der «Graben» zwischen Wirtschaft und Pädagogik beim Thema «Wissensmanagement und Mediendidaktik» kaum kleiner wird, auch wenn es - wie beschrieben - erste Annäherungen und gute Gründe dafür von beiden Seiten gibt. Das wohl wichtigste Verbindungsglied ist das ELearning: Das Interessante dabei ist, dass die Wirtschaft über ein grosses und in vielen Aspekten auch wirksames «Arsenal» an technischen Plattformen und Tools verfügt, aber über nur wenig mediendidaktisches Knowhow. An den Hochschulen ist die Situation vielerorts umgekehrt: An
Konzepten und Methoden für eine didaktisch sinnvolle Gestaltung medialer Lernumgebungen herrscht an den dafür zuständigen Lehrstühlen kein Mangel, wohl aber an der technischen Ausstattung. Wenn der eine Topf und Feuerstelle und der andere eine kalte Suppe hat, zögert man normalerweise nicht, ein naheliegendes Tauschgeschäft $\mathrm{zu}$ machen. «Echte» Kooperationen zwischen Hochschule und Industrie zur Verbesserung von E-Learning, Wissensmanagement und anderen Formen des Wissens und Lernens mit neuen Medien aber sind nach wie vor selten - die Gründe dafür dürften tiefer liegen als in unterschiedlichen Begriffswelten. Doch es gibt Hoffnung, dass es sich ändert: Die mangelnde Akzeptanz von E-Learning und Wissensmanagement in Unternehmen zwingt auch ökonomisch zum Umdenken; eine lernerorientierte Mediendidaktik für die Aus- und Weiterbildung rückt damit in nähere Zukunft. Wie diese Zukunft aussehen könnte, lässt sich nicht konkret vorhersagen. Die beiden Orientierungsmodelle zu Wissensmanagement und E-Learning aus einer pädagogisch-psychologischen Perspektive können aber als Handwerkszeug dafür dienen, nicht mehr nur auf neue Entwicklungen zu reagieren, sondern auch in die Entwicklung neuer Wissens- und Lernmedien gestaltend einzugreifen. Dass dies nicht gegen, sondern mit der Wirtschaft erfolgen sollte, dürfte aus der Argumentation in diesem Artikel hervorgegangen sein. Nur durch faire Kooperationen zwischen wirtschaftlichen und pädagogischen Partnern kann sich auch die Mediendidaktik die Position erarbeiten, die ihr für die Zukunft des Wissens und Lernens mit neuen Medien in allen Kontexten zukommen sollte.

${ }^{8}$ siehe ebenfalls: http://www.netzwerk-medienschulen.de 


\section{Literatur}

Aufenanger, S. (1999). Multimedia und Medienkompetenz - Forderungen an die Medienpädagogik. Sonderheft des GEW-Kreisverbandes Fulda, 5-29.

Back, A. (2002). E-Learning und Wissensmanagement zusammenführen. In: A. Hohenstein \& K. Wilbers (Hrsg.), Handbuch E-Learning (Kap. 7.1). Köln: Deutscher Wirtschaftsdienst.

Back, A., Bendel, O. \& Stoller-Schai, D. (2001). E-Learning im Unternehmen: Grundlagen - Strategien - Methoden - Technologien. Zürich: Orell Füssli.

Back, A., Seufert, S. \& Kramhöller, S. (1998). Technology enabled Management Education: Die Lernumgebung MBE Genius im Bereich Executive Study an der Universität St. Gallen. io management, 3, 36-42.

Bader, R. (2001). Learning Communities im Internet: Aneignung von Netzkompetenz als gemeinschaftliche Praxis. Eine Fallstudie in der pädagogischen Weiterbildung. Münster: LIT.

Bielaczyc, K. \& Collins, A. (1999). Learning communities in classrooms: A reconceptualization of educational practice. In: C. M. Reigeluth (Ed.), Instructional design theories and models (Vol. II) (pp. 269-291). Mahwah, NJ: Erlbaum.

Bullinger, H.-J., Wörner, K. \& Prieto, J. (1998). Wissensmanagement Modelle und Strategien für die Praxis. In: H. D. Bürgel (Hrsg.), Wissens management: Schritte zum intelligenten Unternehmen (S. 21-39). Berlin: Springer.

Eckert, N. (1998). Unternehmensentwicklung und Ökologie des Wissens: Der Primat impliziten Wissens und seine Implikationen für eine evolutionäre Organisationstheorie. Herrsching: Barbara Kirsch.

Friedrich, H. F. \& Mandl H. (1997). Analyse und Förderung selbstgesteuerten Lernens. In: F. E. Weinert \& H. Mandl (Hrsg.), Enzyklopädie der Psychologie, D/I/4, Psychologie der Erwachsenenbildung (S. 273-293). Göttingen: Hogrefe.

Issing, L.J. \& Klimsa, P. (Hrsg.) (2002). Information und Lernen mit Multimedia und Internet. Weinheim: BeltzPVU.

Issing, L.J. (2002). Instruktions-Design für Multimedia. In: L.J. Issing \& P. Klimsa (Hrsg.), Information und Lernen mit Multimedia und Internet (S. 151- 176). Weinheim: Beltz PVU.

Kempfert, G. \& Rolff, H.-G. (1999). Pädagogische Qualitätsentwicklung. Ein Arbeitsbuch für Schule und Unterricht. Weinheim: Beltz.
Kerres, M. (2001). Multimediale und telemediale Lernumgebungen. Konzeption und Entwicklung. München: Oldenbourg.

Kron, F. W. (1993). Grundwissen Didaktik. München: Reinhardt.

Leutner, D. (2002). Adaptivität und Adaptierbarkeit multimedialer Lehrund Informationssysteme. In: L. J. Issing \& P. Klimsa (Hrsg.), Information und Lernen mit Multimedia und Internet (S. 115-125). Weinheim: Beltz PVU.

Müller-Böling, D. (2001). «Uni-www.ersity.de: Lehren und Lernen im Cyberspace». WechselWirkung, Nov./Dez., 20-31.

North, K. (1998). Wissensorientierte Unternehmensführung. Wertschöpfung durch Wissen. Wiesbaden: Gabler.

Polanyi, M. (1966). The tacit dimension. New York: Anchor Day Books.

Probst, G., Raub, S. \& Romhardt, K. (2000). Wissen managen. Wie Unternehmen ihre wertvollste Ressource optimal nutzen. Frankfurt am Main; Wiesbaden: FAZ/ Gabler.

Reinmann-Rothmeier, G. \& Mandl, H. (1997). Wissensmanagement in der Schule. Profil, 10, 20-27.

Reinmann-Rothmeier, G. \& Mandl, H. (2000). Ein pädagogisch-psychologischer Ansatz zum Wissensmanagement: Ein Widerspruch in sich? io management, 11, 68-75.

Reinmann-Rothmeier, G. \& Mandl, H. (2002). Analyse und Förderung kooperativen Lernens in netzbasierten Umgebungen. Zeitschrift für Entwicklungspsychologie und Pädagogische Psychologie, 34 (1), 44-57.

Reinmann-Rothmeier, G. \& Mandl, H. (Hrsg.) (2001). Virtuelle Seminare in Hochschule und Weiterbildung: Drei Beispiele aus der Praxis. Bern: Huber.

Reinmann-Rothmeier, G. (2001). Münchener Modell: Eine integrative Sicht auf das Managen von Wissen. Wissensmanagement, 5, 51-54.

Reinmann-Rothmeier, G., Mandl, H., Erlach, C. \& Neubauer, A. (2001). Wissensmanagement lernen. Weinheim: Beltz.

Renkl, A. (1996). Träges Wissen: Wenn Erlerntes nicht genutzt wird. Psychologische Rundschau, 47, 62-78.

Romhardt, K. (2002). Wissensgemeinschaften: Orte lebendigen Wissensmanagements. Dynamik - Entwicklung - Gestaltungsmöglichkeiten. Zürich: Versus.

Schlaffke, W. (1999). Qualität und internationale Wettbewerbsfähigkeit Der Beitrag der betrieblichen Weiterbildung. In: R. Tippelt (Hrsg.), 
MedienPädagogik

Handbuch Erwachsenenbildung, Weiterbildung (S. 478-486). Opladen: Leske + Budrich.

Schneider, U. (2001). Die 7 Todsünden im Wissensmanagement: Kardinaltugenden für die Wissensökonomie. Frankfurt/M.: FAZ Verlagsbereich Buch.

Schnotz, W. (2002). Wissenserwerb mit Texten, Bildern und Diagrammen. In: L. J. Issing \& P. Klimsa (Hrsg.), Information und Lernen mit Multimedia und Internet (S. 65 - 81). Weinheim: Beltz PVU.

Schott, F. (1991). Instruktionsdesign, Instruktionstheorie und Wissensdesign: Aufgabenstellung, gegenwärtiger Stand und zukünftige Herausforderung. Unterrichtswissenschaft, 19, 195-217.

Schulz-Zander, R. (2001). Neue Medien als Bestandteil von Schulentwicklung. In: S. Aufenanger, R. Schulz-Zander \& D. Spanhel (Hrsg.), Jahrbuch Medienpädagogik 1 (S. 263-282). Opladen: Leske und Budrich.

Schüppel, J. (1996). Wissensmanagement. Wiesbaden: Deutscher Universitäts Verlag.

Senge, P. M., Kleiner, A., Smith, B., Roberts, C. \& Ross, R. (1997). Das Fieldbook zur Fünften Disziplin. Stuttgart: Klett-Cotta.

Weidenmann, B. (2002). Abbilder in Multimediaanwendungen. In: L. J. Issing \& P. Klimsa (Hrsg.), Information und Lernen mit Multimedia und Internet (S. 83 - 96). Weinheim: Beltz PVU.

Wilbers, K. (2002). E-Learning didaktisch gestalten. In: A. Hohenstein \& K. Wilbers (Hrsg.), Handbuch E-Learning (Kap. 4.0). Köln: Deutscher Wirtschaftsdienst.

Willke, H. (1998). Systemisches Wissensmanagement. Stuttgart: Lucius und Lucius. 